\title{
DIGITAL MATERIAL REPRESENTATION MODEL OF POROUS MICROSTRUCTURE BASED ON 3D RECONSTRUCTION ALGORITHM
}

\begin{abstract}
Development of the Digital Material Representation (DMR) model, based on 3D reconstruction algorithm and serial sectioning, is the main goal of the present paper. Details on the serial sectioning and image processing algorithms are presented first. Serial sectioning is realized on the basis of light microscopy (LM). Then concept of 3D reconstruction and developed algorithms are presented. Two approaches, based on shape coefficients and the flood fill algorithms, are developed to identify corresponding features on subsequent 2D images. Then, the interpolation algorithm to reconstruct 3D volume between 2D images is presented. Finally, obtained 3D model is an input for finite element mesh generation software for subsequent finite element calculations.

Keywords: 3D reconstruction, digital material representation, serial sectioning, porous microstructure, finite element method
\end{abstract}

\section{Introduction}

The sintered porous materials become increasingly popular in various practical applications (e.g. bearing materials, tool materials, super hard cutting materials etc.) because they provide desired physical and chemical properties of products. They also give the opportunity to obtain products with a specific microstructure, porosity and permeability. However, to improve and control material properties, like tensile strength, a detailed knowledge on influence of porous microstructure on material behaviour under deformation conditions is of importance. Forming processes of porous metallic materials are characterised by a complex spatial state of stress and/or deformation. Due to this three-dimensional nature of plastic deformation, analysis of material behaviour, that is based only on two-dimensional metallographic images, is often insufficient.

In that case, application of numerical modelling techniques can support and extend three dimensional experimental investigation performed on this group of materials. However, the key aspect in development of reliable and robust numerical simulation is to apply accurate rheological model, which takes into account all major mechanisms controlling material behaviour under plastic deformation. In the paper, an approach to develop Digital Material Representation 3D model, based on reconstruction algorithm of $2 \mathrm{D}$ images of investigated porous microstructure, is presented.

\section{Digital Material Representation concept}

The concept of Digital Material Representation (DMR) assumes that each important feature of the microstructure is explicitly taken into account during numerical simulations. Thus, both shape as well as properties of e.g. grains, phases, inclusions, precipitates etc. can be considered and in detail investigated during material deformation, like in the real microstructure $[1,2]$.

There are various ways of obtaining initial morphology of investigated microstructures (see [1] for details). However, the most accurate approach is based on image processing methods applied to images of real microstructure. An electron microscopy images can be used as an input data. Additionally, if e.g. electron backscatter diffraction (EBSD) detector is employed, then not only information regarding microstructure geometry is obtained but also information about initial crystallographic orientation is provided $[3,4]$. Unfortunately, the approach is time consuming and expensive, because each numerical simulation, based on DMR, requires such an EBSD analysis. That is why, image processing is most often applied to the light microscopy images, which are much more affordable. However, in this case, only information regarding grain morphology can be obtained.

Obtaining 3D digital representations, based on the experimental data, is even more demanding. In this case advanced experimental procedures have to be used, e.g. near-field high-energy $\mathrm{X}$-ray diffraction microscopy (nf-HEDM) or X-ray diffraction contrast tomography. Both methods are classified as non-destructive approaches, thus the microstructure can be investigated prior and after deformation. However, these methods are still highly

\footnotetext{
* AGH UNIVERSITY OF SCIENCE AND TECHNOLOGY, AL. A. MICKIEWICZA 30, 30-059 KRAKOW, POLAND

** SILESIAN UNIVERSITY OF TECHNOLOGY INSTITUTE OF MATERIALS SCIENCE, FACULTY OF MATERIALS ENGINEERING AND METALLURGY, 8 KRASIŃSKIEGO STR., 40-019 KATOWICE, POLAND

\# Corresponding author: 1.madej@agh.edu.pl
} 
expensive, and the volume of investigated sample is limited. That is why, the DMR in 3D cases is usually created based on the reconstructed 2D slices obtained using a destructive method - the serial sectioning. Again, a light or scanning electron microscopy (SEM) can be used during the serial sectioning procedure to provide input data for image processing and reconstruction algorithms.

The conventional approach to serial sectioning, based on manual labour, is extremely time consuming and requires a series of steps. First one involves very precise polishing. The sample is polished for a required time to remove desired amount of material. Then, the sample is subjected to rinsing and etching stages. During the last stage, image of the microstructure is acquired in a light microscopy equipped with digital camera. This procedure is repeated desired number of times until proper volume of material is analysed.

If, besides the grain shape, also crystallographic orientation is required, then a series of $2 \mathrm{D}$ images from the scanning electron microscopy have to be acquired. The serial sectioning in this case can be realized in semi-automated manner by a SEM/FIB/EBSD technique. The focused ion beam (FIB) can be used to remove atoms from the analysed surface, and then a 2D EBSD map acquisition can take place. This procedure is performed in subsequent manner and eventually results in revealing microstructure from increasing depths of the sample. The limitation of the method is related to relatively small areas of material that can be investigated.

In both cases of serial sectioning, after 2D images acquisition, additional image processing and reconstruction procedures have to be applied to obtain 3D microstructure representation as shown in Fig. 1.

The key elements in the presented approach are precise polishing operations and development of accurate reconstruction algorithms, to obtain a full $3 \mathrm{D}$ representation of the investigated volume of material. These two stages are off importance, during creation of DRM model for further finite element simulations.
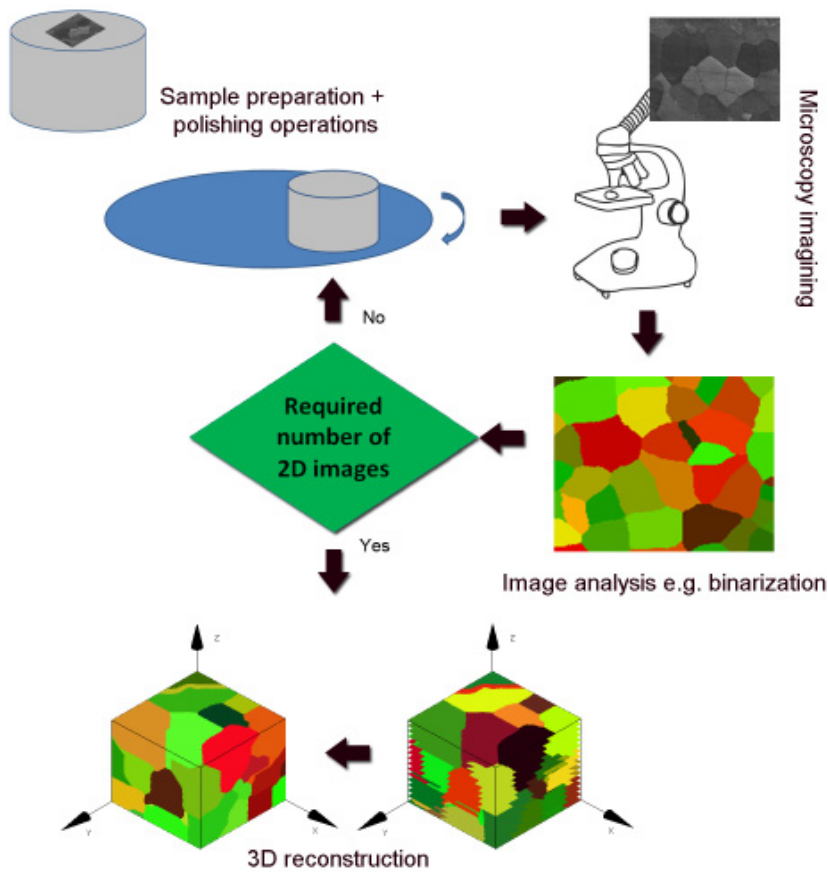

Fig. 1. Concept of the serial sectioning approach
Development of mentioned 3D reconstruction algorithm, dedicated for porous microstructures, was the main goal of the present work. The serial sectioning, based on light microscopy analysis, was selected for the investigation as only this approach provided sufficient dimensions of material area for further investigation.

\section{Image processing}

As mentioned, manual serial sectioning was realised to provide input data for the $3 \mathrm{D}$ reconstruction algorithm. The procedure consisted of several steps: precise vibration polishing, microhardness indentation for marking the area of interest, acquisition of high resolution digital images of microstructure observed under light microscopy, and finally, subsequent image processing operations to prepare data for $3 \mathrm{D}$ reconstruction.

The size of the pores in the investigated microstructure determined a layer thickness, which was removed during single polishing operation. Because the smallest pore diameters in the sample were approx. 1-2 $\mu \mathrm{m}$, the thickness of removed layer was set to $0.5 \mu \mathrm{m}$ to provide at least 2-3 slices across each pore. On the basis of the preliminary experiments, two parameters of polishing were determined: load applied to the samples $-610 \mathrm{~g}$, and polishing time -12 minutes. The water suspension of $\mathrm{Al}_{2} \mathrm{O}_{3}$ $(0.05 \mu \mathrm{m}$ particle size) was used during this process. After first polishing stage, the area of interest was marked by two microhardness indentations to identify the investigated region and evaluate the thickness of removed layers (Fig. 2).

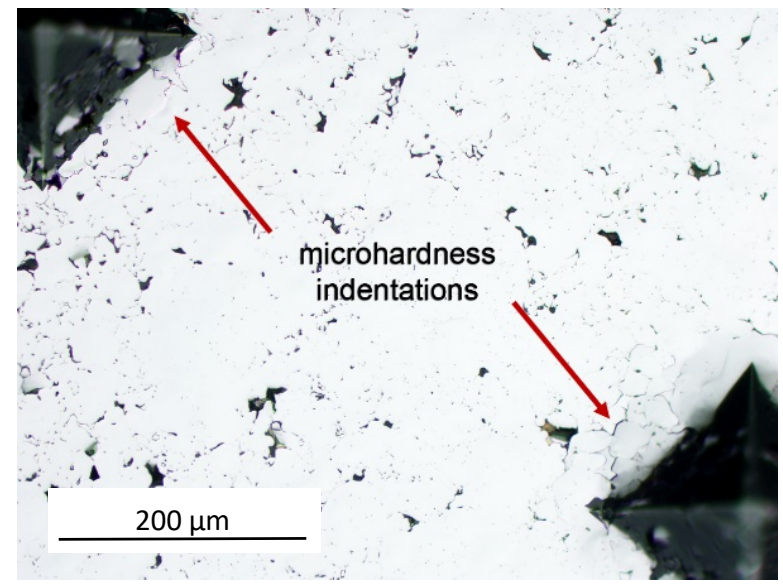

Fig. 2. Location of microharndess indents used in serial sectioning procedure

The microstructure observations of unetched, polished cross-sections of the specimens were performed in bright field on Olympus GX71 light microscope with DP70 digital camera. The specimens were preliminary adjusted on the basis of microhardness indentation positions and images of the microstructure were captured at $4080 \times 3072$ resolution. During the procedure, a stack of 21 images of parallel cross-sections of porous microstructure was obtained and used as input data for further processing. 
The proposed algorithm, responsible for transferring acquired LM images into the DMR form, consists of several subsequent steps. First, microstructure images were precisely adjusted within the stack using correlation between grey levels and marked, relevant points in subsequent images. Then, images were subjected to digital treatment with the threshold function. This step was realised in the Metllo software [5]. The thresholding algorithm performs simple binarisation procedure. Grey pixels represent pixels of the image, which values are below the thresh- old range, and black pixels represent pixels with values higher than the threshold range. After thresholding, some noise can be observed in the image, which disrupts visual separation of the two phases. To remove that noise the filtering algorithm was applied. Finally, small artificial features (area below 7 pixels) were eliminated to obtain representation of matrix with pores (Fig. 3).

Obtained stack of 2D binary images of subsequent layers of investigated microstructure were an input for the developed reconstruction algorithm. a)

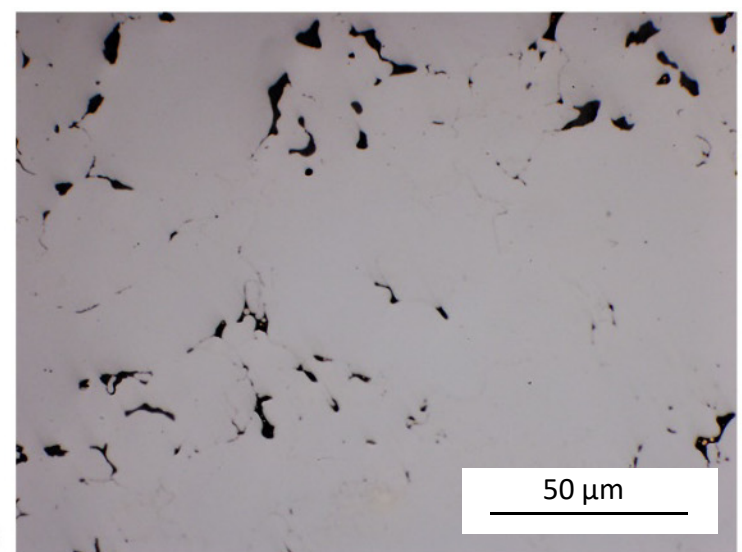

b)

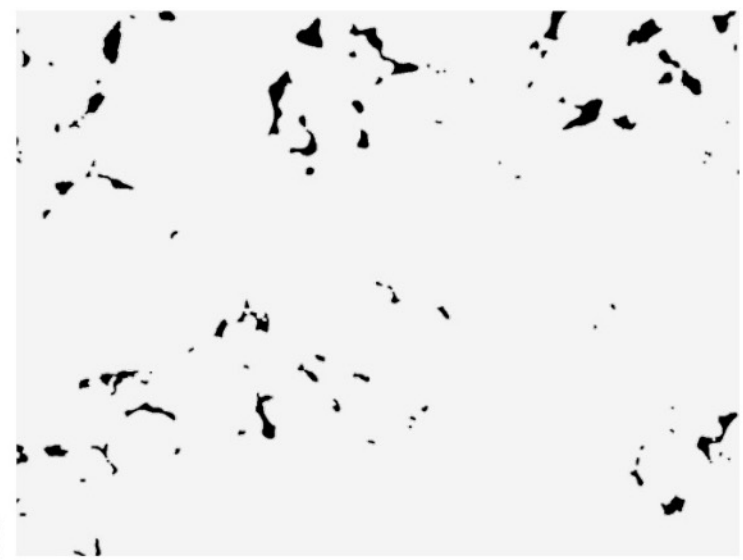

Fig. 3. Example of the: a) structure and b) digital representation of sintered porous microstructure

\section{Algorithm for 3D reconstruction}

As presented, after the serial sectioning procedure, a set of two dimensional images was obtained. The physical distance between subsequent images in the analysed case study was equal to $0.5 \mu \mathrm{m}$. In order to create a DMR model for further finite element simulations, the space between subsequent $2 \mathrm{D}$ slices has to be filled to fully reconstruct 3D volume. To do that, authors proposed an efficient algorithm designed to reconstruct microstructure of porous material.

The key step in the procedure is proper identification of corresponding features (pores) on two subsequent images, as schematically presented in Fig. 4

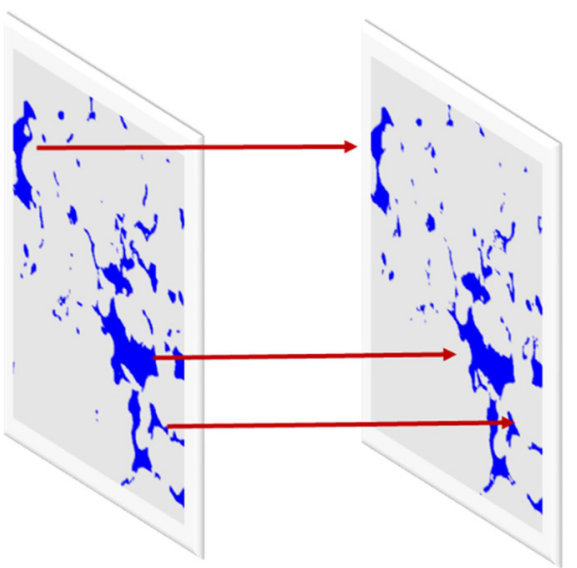

Fig. 4. Schematic illustration of identification of corresponding features on two subsequent images
Two different algorithms were developed to automatically identify corresponding features (pores). First one is based on evaluation and comparing shape coefficients of subsequent features [1,6], and the second one is based on the flood fill algorithm [7].

In the first approach, the set of standard shape coefficient, proposed in [6], was used, namely:

- circularity coefficient:

$$
\xi=2 \sqrt{\frac{S}{\pi}}
$$

- $\quad$ second circularity coefficient:

$$
\xi=\frac{L}{\pi}
$$

- Malinowska coefficient

$$
\xi=\frac{L}{2 \sqrt{\pi S}}-1
$$

- Blair-Bliss coefficient:

$$
\xi=\frac{S}{2 \sqrt{\pi S}}-1
$$

- $\quad$ ratio between the minimum $\left(r_{\min }\right)$ and maximum $\left(r_{\max }\right)$ distance between contour and the center of gravity of investigated feature:

$$
\xi=\frac{r_{\min }}{r_{\max }}
$$

where: $S, L-$ area and perimeter of an analysed feature, respectively. 
Calculated shape coefficients from the first image were compared for each feature with the set of coefficients from the second 2D image. Features with the smallest difference in shape coefficients were grouped and the same identification number (id number) was assigned.

As shapes of pores are highly irregular, application of shape coefficients in some cases does not provide satisfactory results. For these situations a flood fill algorithm was implemented. To reduce memory usage as well as to shorten execution time, non-recurrent version was used. As the input images were in a binary form, it was assumed that each pixel/cell was represented by one of two values: 1 for cells belonging to a feature (pore) and 0 for the matrix material. The algorithm simply compares corresponding pixels from two slices and if two pixels with the same coordinates from two subsequent slices have the id value of 1 , then the solution assumes that the space between these two pixels will be interpolated (Fig. 5). Since the distance between each slice was small, the implementation based on flood fill provided accurate reconstruction and supplements mentioned algorithm based on shape coefficients.

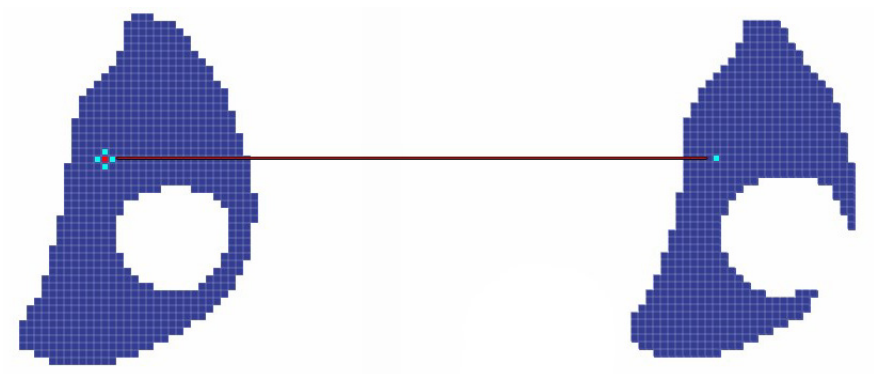

Feature from slice 1

Feature from slice 2

Fig. 5. Illustration of the flood fill algorithm concept

Eventually, particular pores on two subsequent images were identified as schematically presented in Fig. 6.
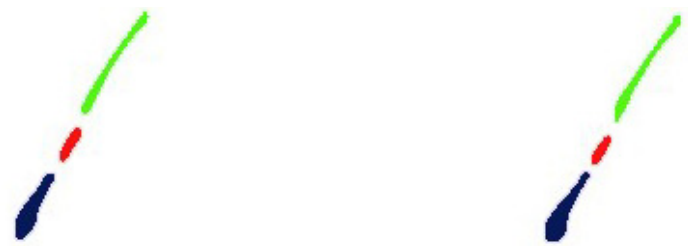

Slice 1

Slice 2

Fig. 6. Illustration of pores with the same id numbers identified on two subsequent images

Each individual features (pore) was represented by a unique id number to allow the algorithm to distinguish different elements during the interpolation stage, which is the basis of the $3 \mathrm{D}$ reconstruction algorithm. During this stage, the algorithm takes pair of slices and proceeds to find set of features sharing same id number, then a new feature can be created based on cells that each feature share with the rest (Fig. 7).

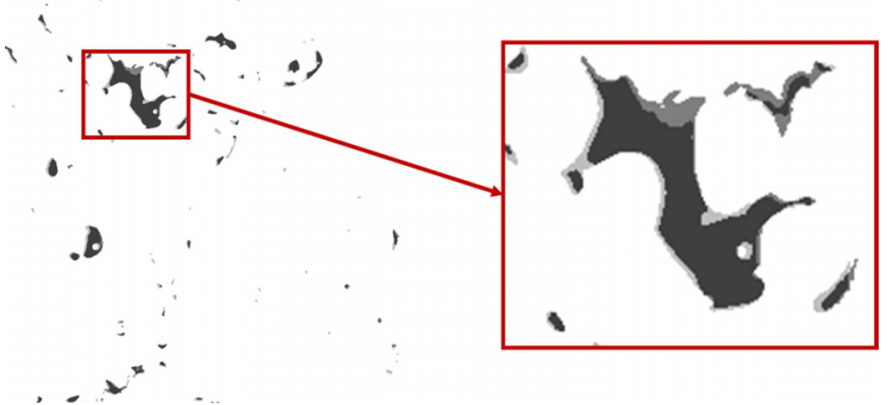

Fig. 7. Two corresponding slices (merged) with shared cells. Black cells are shared parts of each feature set, grey cells belong only to one feature

Prior interpolation, cells located along the shared features (pores) boundaries have to be identified. Features edge consists of cells, which have at least one neighbour with id equal to 0 , representing solid matrix. In order to identify these boundary cells, algorithm checks each neighbour of investigated cell and if the mentioned condition is met, adds the cell to the edge cells list. The concept of the proposed algorithm is illustrated in Fig. 8.

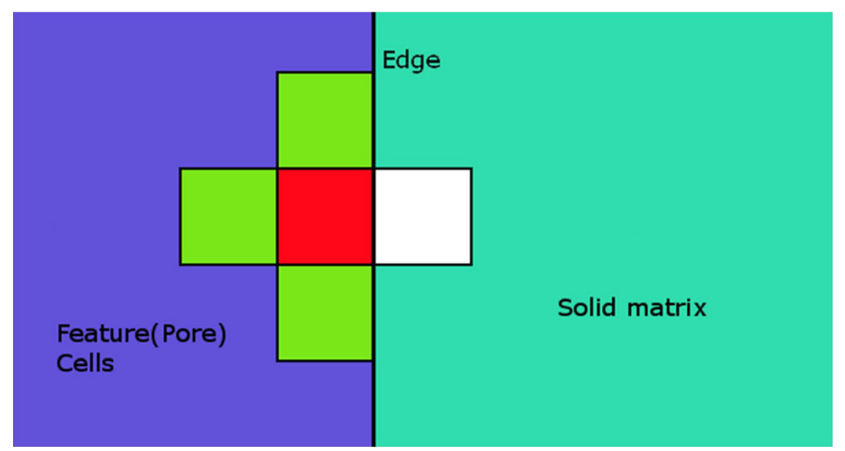

Fig. 8. Edge finding algorithm

Red cell in Fig. 8 represents the investigated cell, green cells are part of the feature (pore) and white cell is located in the area of the matrix. As seen in Fig. 8, the cell marked by the red colour has one neighbour that is not part of a feature, which means that the cell is located along the feature boundary. The algorithm is usually realised in an iterative manner until all cells at the particular image are analysed. As a result, all cells along the feature (pore) boundary can be identified (Fig. 9).
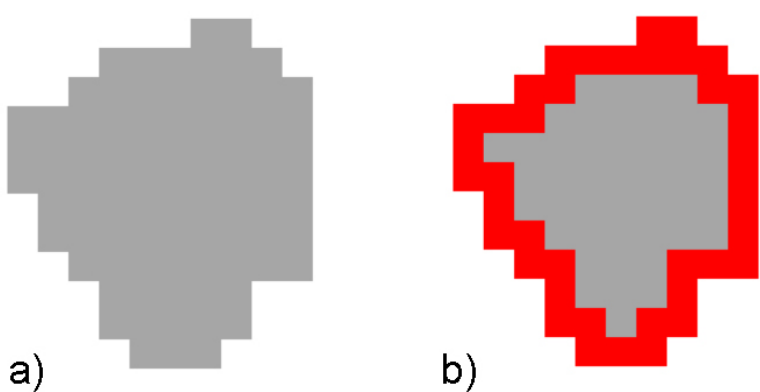

Fig. 9. Example of a feature (pore) shape: a) without and b) with marked edges 
In order to reduce required memory space, during the interpolation, only information on cells belonging to features (pores) were stored. First, cells with positions exactly corresponding at two consecutive slices were extruded, as seen in Fig. 10. Then, positions for other sections of the feature were interpolated between two slices based on the following set equations:

$$
\left\{\begin{array}{l}
x_{i}=x_{1} \frac{i}{N}+x_{n}\left(1-\frac{i}{N}\right) \\
y_{i}=y_{1} \frac{i}{N}+y_{n}\left(1-\frac{i}{N}\right) \\
z_{i}=i
\end{array}\right.
$$

where: $i$ - number of currently interpolated slice, $N$ - total number of interpolated slices between the two investigated ones, $x$, $y, z$ - cell coordinates.

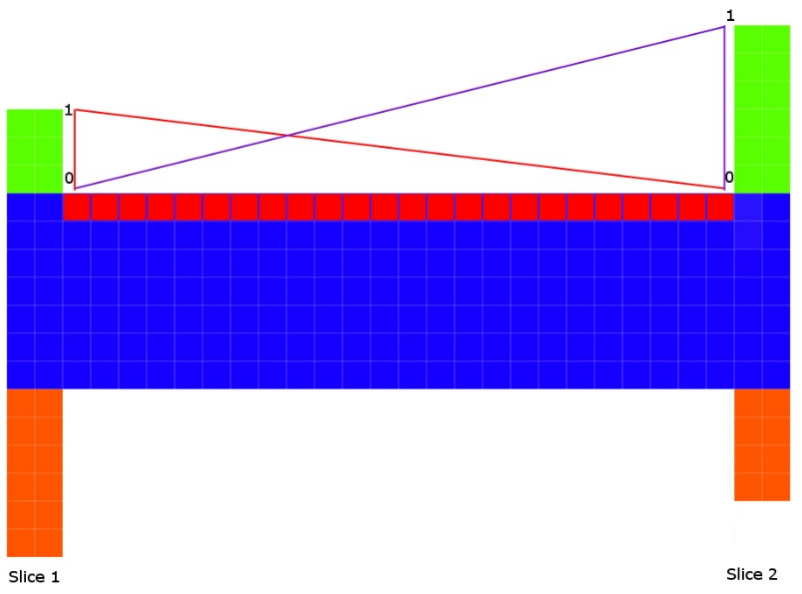

Fig. 10. Visual presentation of interpolation process of a single feature between two investigated slices (blue colour represents cells that have exactly the same $x$ and $y$ coordinates at two investigated slices, green and orange represents cell that will be used during interpolation)
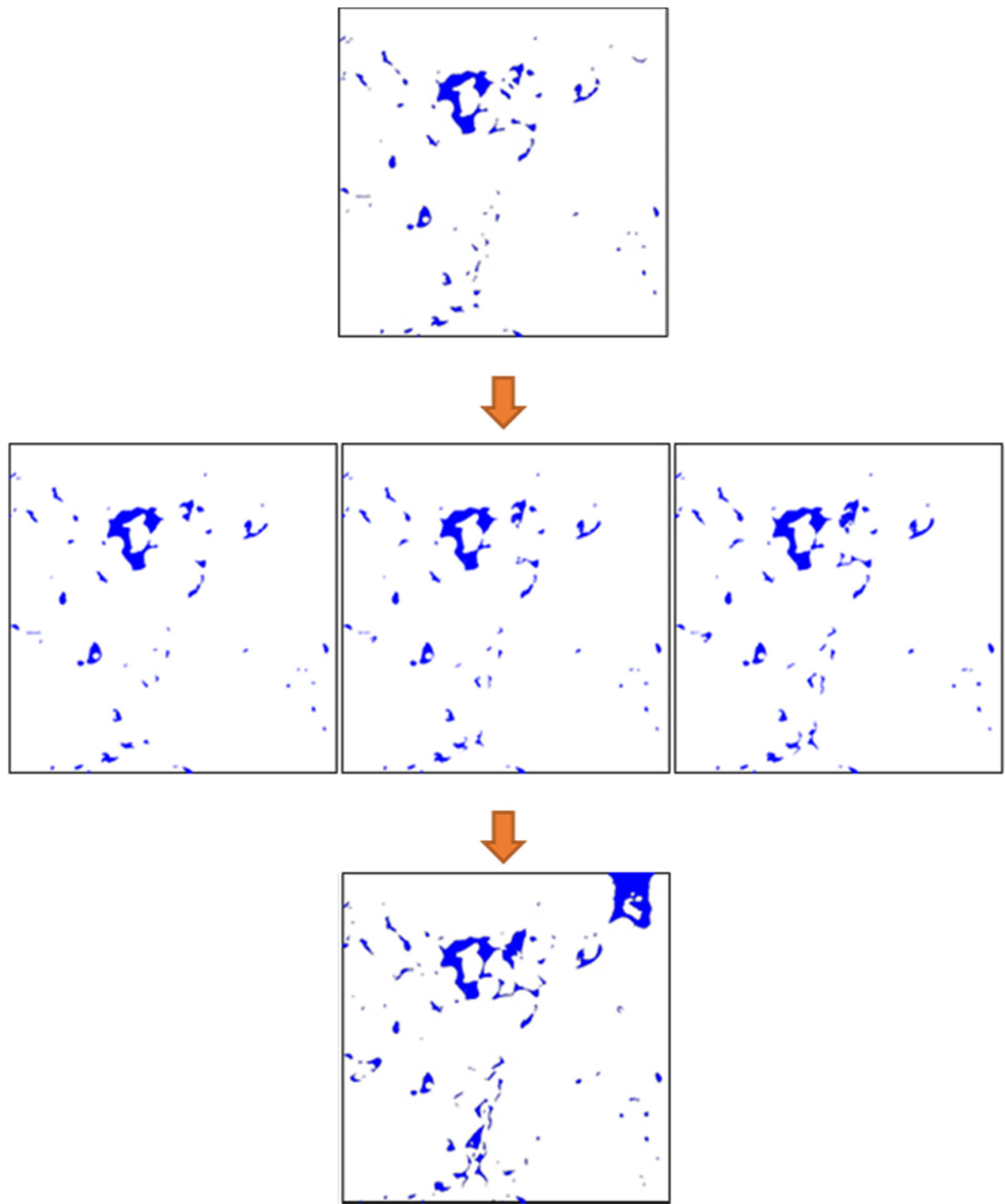

Fig. 11. Two input images obtained from LM and set of 3 reconstructed images between them 


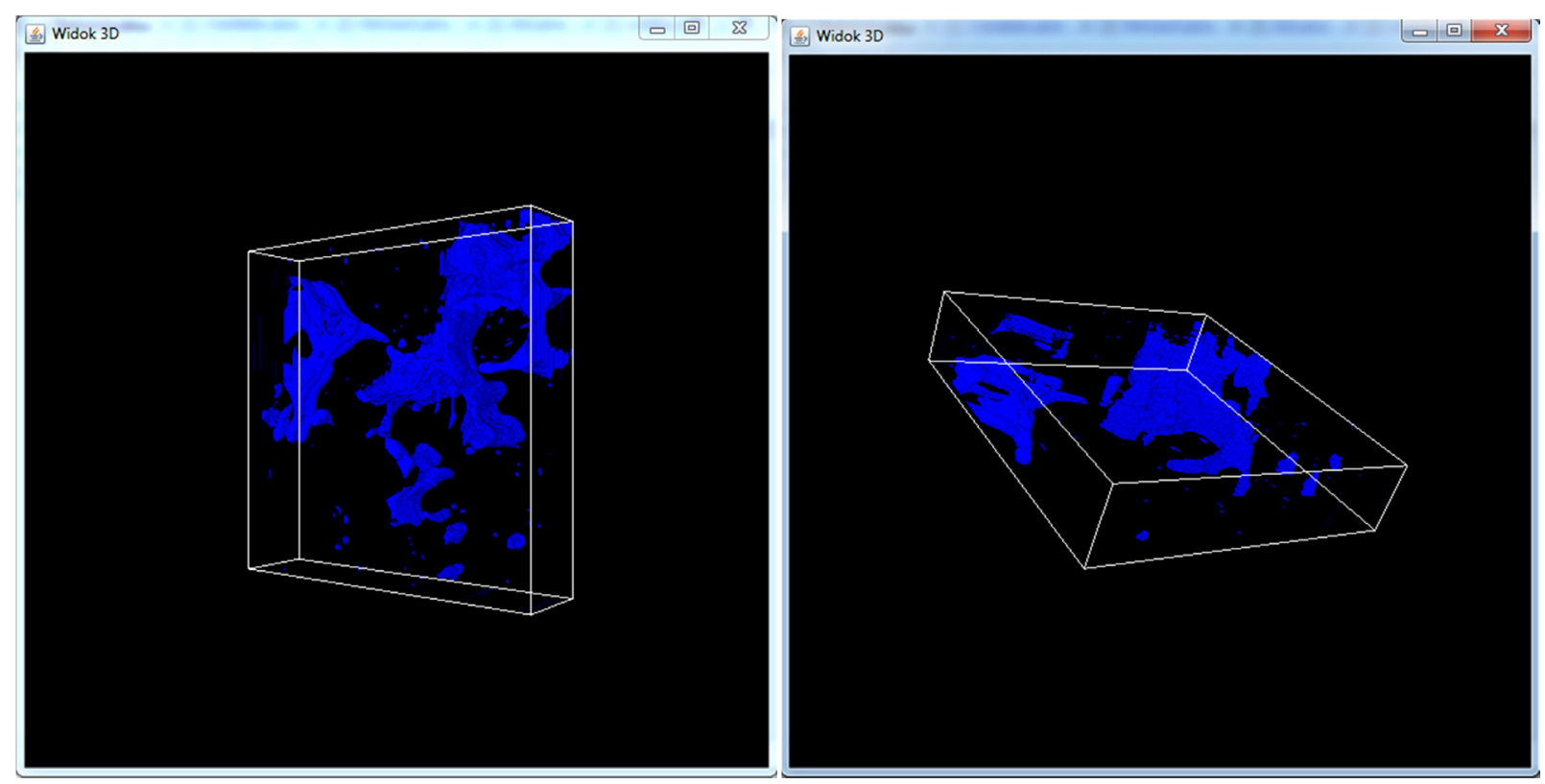

Fig. 12. Reconstructed volume of investigated porous microstructure from serial sectioning operations with pores marked by blue colour. Physical size of the sample $44 \times 44 \times 10.5 \mu \mathrm{m}$

Developed algorithm have to be applied separately to each pair of 2D images obtained from serial sectioning procedure and recreates 2D images between them (Fig. 11), thus as a result, fully $3 \mathrm{D}$ reconstruction can be performed as presented in Fig. 12.

Information provided from the reconstructed $3 \mathrm{D}$ digital microstructure regarding position of pores within the material was an input for the finite element mesh generation algorithm. Additionally, to properly capture in homogeneities in FE calculations along the pores, generated FE meshes were refined along these regions, as seen in Fig. 13. The mesh generation algorithm is based on classical Delannay triangulation, with specific algorithms responsible for refinement of elements along the grain/ phase interfaces [8].

The DMR model with generated mesh (Fig. 13), can be finally used during numerical investigation of material behaviour under various loading conditions to evaluate influence of pores on final properties.

\section{Conclusions}

The main goal of the paper was development of 3D reconstruction algorithm based on input data from serial sectioning procedure. Based on the presented research, several conclusions can be drawn:

- $\quad$ application of vibration polishing, to reveal subsequent layers of the investigated material, provides sufficient control and accuracy in case of the removal depth,

- shape of microhardness indentation marks is a good indicator for calculations of amount of removed material,

- implementation of shape coefficients to distinguish matching features on two subsequent images seems to be an effective solution,

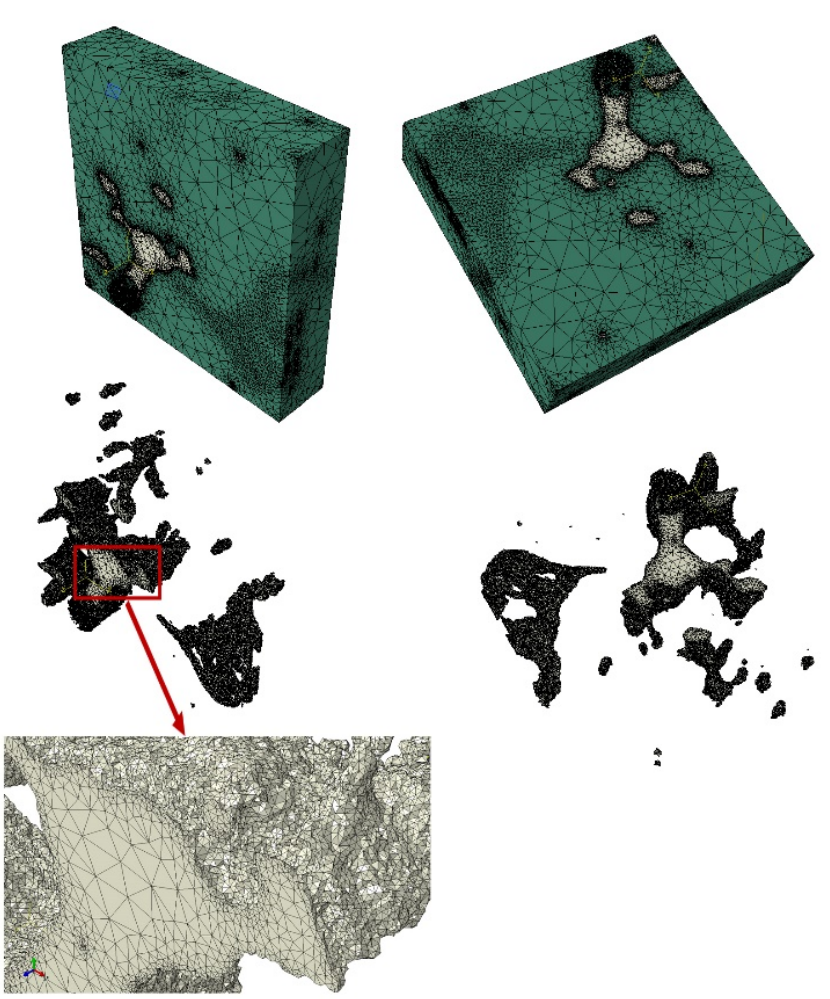

Fig. 13. Digital Material Representation model of 3D reconstructed volume (view from two different angles) with the FE mesh for subsequent numerical simulations. Pores are also presented separately to highlight their complex shapes

- the flood fill algorithm is also an effective solution in pores identification in case when subsequent images are in close proximity,

- $\quad$ application of simple interpolation algorithm for the reconstruction purposes provides fully 3D information on microstructure morphology for FE mesh generation. 
Developed DMR will be used in the future work to evaluate pores behaviour under complex loading conditions involving strain patch changes.

\section{Acknowledgement}

Financial assistance of the National Science Center project No. 2014/15/B/ ST8/00086 is acknowledged.

\section{REFERENCES}

[1] M. Pietrzyk, L. Madej, L. Rauch, D. Szeliga, Computational Materials Engineering: achieving high accuracy and efficiency in metals processing simulations, 2015 Butterworth-Heinemann Elsevier.
[2] O.N. Senkov, D.B. Miracle, S.A. Firstov, Metalic materials with high structural efficiency, NATO Science Series 146 (2003).

[3] D. Raabe, R.C. Becker, Model. Simul. Mater. Sc. 8, 445-462 (2000).

[4] R. Logé, H. Resk, Z. Sun, L. Delannay, M. Bernacki, Steel Res. Int. 81, 1420-1425 (2010).

[5] L. Wojnar, K.J. Kurzydłowski, J. Szala, ASM Handbook Metallography and Microstructures 9, 403-427 (2004).

[6] J. Schroeder, D. Balzani, D. Brands, Arch. Appl. Mech. 81, 975979 (2011).

[7] www.crbond.com (2011).

[8] M. Madej, F. Krużel, P. Cybułka, K. Perzyński, K. Banaś, Computer Methods in Materials Science 12, 85-96 (2012). 